\title{
Microscopic Theory of Damon-Eshbach Modes in Ferromagnetic Films
}

\author{
R. V. Leite and R. N. Costa Filho \\ Departamento de Física, Universidade Federal do Ceará, Caixa Postal 6030 \\ Campus do Pici, 60451-970 Fortaleza, Ceará, Brazil \\ E-mail: rai@fisica.ufc.br, FAX:+-55-85-2889903
}

\begin{abstract}
The surface spin wave branches in ferromagnetic films are studied using a microscopic theory which considers both magnetic dipole-dipole and Heisenberg exchange interactions. The dipole terms are expressed in a Hamiltonian formalism, and the dipole sums are calculated in a rapidly convergent form. The Damon-Eshbach surface modes are analyzed for different directions of the spin-wave propagation and also for different ratios of the strength of the dipole interactions relative to the exchange interactions. Numerical results are presented using parameters for $\mathrm{Fe}$ and $\mathrm{GdCl}_{3}$.
\end{abstract}

Keywords: Ferromagnetic Films, Spin Waves, Magnons, Dipolar Interactions. 
It is well known theoretically that, in quasi-two-dimensional systems, such as ultrathin ferromagnetic films, the short-range exchange interactions alone are not necessarily sufficient to establish a Ferromagnetic ordering above the ground state, and that it is necessary to take into account the anisotropy and long-range character of the dipolar interactions [1]. Regarding the spin dynamics in ferromagnetic films, a macroscopic (or continuous medium) theory for the dipole-dominated regime was given by Damon and Eshbach [2] in terms of magnetostatic modes. They identified a surface branch of the spectrum, now known as the Damon-Eshbach (DE) mode. Recently, microscopic theories have been used to study the dipole-exchange spin waves (SW) in ferromagnetic and antiferromagnetic films [3] [5].

The DE mode has a number of striking and unusual properties. For example, the existence condition of this mode depends on the propagation angle relative to the principal axes of the crystal. The x-direction in our case, being within the range $0 \ll \theta<\theta_{c}$, where $\theta_{c}$ depends on the characteristics of the system, such as the applied field and dipolar strength (see Ref. 6 for a review). The aim of this paper is to study the DE modes in ferromagnetic films at low temperatures $\left(T \ll T_{c}\right.$, where $T_{c}$ is the Curie temperature) by using a microscopic theory, and including both magnetic dipole-dipole and Heisenberg exchange interactions. The dipole terms are expressed in a Hamiltonian formalism, and the dipole sums are calculated in a rapidly convergent form. In order to find the critical $\theta_{c}$ where the DE modes vanish, we have to study the dependence of the DE modes and the SW energies (for each of the discrete branches) on the variation of the exchange parameter, on the strength of the dipolar interaction, and on the wave vector direction of propagation. Another important factor in the analisys is the effect of different dipole interaction strengths (relative to the exchange)on the dispersion relation of the SW. Numerical results are presented using parameters for Fe and $\mathrm{GdCl}_{3}$.

Consider a ferromagnetic film with $N$ atomic layers arranged on a simple cubic lattice, with lattice constant $d$. The wave vector $\mathbf{q}$ makes an angle $\theta$ with respect to the $x$-axis, and the Zeeman field $H_{0}$ is assumed to be parallel to $z$ direction. The surface of the film is in the $x-z$ plane, while the $y$-axis is perpendicular to the film. The Hamiltonian can be written 
as

$$
H=-\frac{1}{2} \sum_{i j} J_{i j} \mathbf{S}_{i} \cdot \mathbf{S}_{j}-g \mu_{B} H_{0} \sum_{i} S_{i}^{z}+\frac{1}{2}\left(g \mu_{B}\right)^{2} \sum_{i j} D_{i j}^{\alpha \beta} S_{i}^{\alpha} S_{j}^{\beta},
$$

where $\mathbf{S}_{i}$ is the spin operator at site $i$ and $J_{i j}$ is the exchange coupling between sites labeled $i$ and $j$. We assume that the exchange coupling is $J_{1}$ and $J_{2}$ for nearest and next-nearest neighbors respectively. The second term of the above equation is the Zeeman term, and the dipole-dipole interaction between spins is represented by the last term, where $\alpha$ and $\beta$ denote the components $x, y$, or $z$, and

$$
D_{i j}^{\alpha \beta}=\left\{\left|\mathbf{r}_{i j}\right|^{2} \delta_{\alpha \beta}-3 r_{i j}^{\alpha} r_{i j}^{\beta}\right\} /\left|\mathbf{r}_{i j}\right|^{5}
$$

whit $\mathbf{r}_{i j}=\mathbf{r}_{j}-\mathbf{r}_{i}$, and the case $i=j$ excluded from the sums in Eq. (1).

Using the Holstein-Primakoff transformations, the Hamiltonian can be expanded as $H=$ $H^{(2)}+H^{(3)}+H^{(4)}+\ldots$ (apart from a constant), where $H^{(m)}$ denotes the term involving a product of $m$ boson operators. Here we are considering the non-interacting (linear) SW modes that are described by the quadratic term $H^{(2)}$. Therefore, the Hamiltonian takes the form:

$$
H=\sum_{q n n^{\prime}}\left\{A_{n n^{\prime}}(\mathbf{q}) a_{q n}^{\dagger} a_{q n^{\prime}}+B_{n n^{\prime}}(\mathbf{q})\left[a_{q n} a_{-q n^{\prime}}+a_{q n}^{\dagger} a_{-q n^{\prime}}^{\dagger}\right]\right\}
$$

Here we are using a representation of the boson operators $a^{\dagger}$ and $a$ in terms of a $2 D$ wave vector $\mathbf{q}=(q \cos \theta, q \sin \theta), \mathbf{q}$ being the modulus of $\mathbf{q}$, parallel to the film surface and indices $n, n^{\prime}(=1,2, \ldots, N)$ that label the atomic layers parallel to the surface. The coefficients $A_{n n^{\prime}}(\mathbf{q})$ and $B_{n n^{\prime}}(\mathbf{q})$ are described in Ref.5. A linear transformation to new boson operators can then be found such that $H$ takes a diagonalized form [9]. Its eingenvalues yield the energies $E_{\nu}(\mathbf{q})$ of the discrete SW branches, where $\nu(=1,2, \ldots, \mathrm{N})$ labels the branches.

In Fig. 1(a), we show the behavior of the lowest SW branches for very small wave vectors in a $\mathrm{GdCl}_{3}$ film with $N=20$. For this material, we use the parameters; $4 \pi M=$ $\left(g \mu_{B} S\right) / d^{3}=0.82 \mathrm{~T}$ to characterize the dipolar strength, the bulk exchange field $H_{E x}=$ $6 S\left(J_{1}+2 J_{2}\right)=0.54 \mathrm{~T}$, and $H_{0}=0.36 \mathrm{~T}$. We have taken the case of $\theta=0$ (the Voigt 
geometry), for which it is known that there is a Damon-Eshbach (DE) surface mode [2] in the magnetostatic continuum limit where exchange is neglected. It can be seen that a purely magnetostatic DE mode starts at $q_{x}=q=0$ at the frequency $18.25 \mathrm{GHz}$. There are hybridizations until the eighth mode, and the DE mode tends to be flat around the value $21.5 \mathrm{GHz}$ with increasing $q_{x}$. On the other hand, in a material such as Fe that has a much smaller value for the ratio $4 \pi M / H_{E x}$, it is necessary to consider a much larger film thickness in order for the DE mode to hybridize with the lowest bulk branch. This is because in a thicker film, the density of modes increases and create more modes in the region where the DE mode propagates, making hybridizations possible. An example for Fe is given in Fig. 1(b) considering $N=120,4 \pi M=2.14 \mathrm{~T}, H_{E x}=2140 \mathrm{~T}$, and $H_{0}=0.054 \mathrm{~T}$. Only the lowest branches of the spectrum are shown in Fig. 1(b), where it can be seen that much smaller values of $q_{x}$ are necessary (compared with the previous example) to see the analogue of the DE mode in the spectrum.

In Fig. 2, we show results for the SW frequencies of the above-mentioned materials considering the SW propagation direction $\theta$. In Fig. 2(a), the dependence of the lowest $\mathrm{GdCl}_{3} \mathrm{SW}$ branches on $\theta$ is shown for a fixed value $q a / \pi$. For small wavevectors, the branches decrease their frequency values as $\theta=0$ goes from 0 to $\theta=\pi / 2$. As in Fig. 1 , the hybridization of the modes is seen to decrease until the second mode. The two lowest branches are the most affected by the SW propagation direction. The others tend to become flat after certain angle values. The figure also shows the reciprocity of the dispersion relation as the angle goes to $\pi$. The same effect can be observed in Fig. 2(b) considering Fe. In this case, the dependence of the modes on the angle is much weaker than in the case of $\mathrm{GdCl}_{3}$, and only the two lowest modes are affected by the propagation angle.

In conclusion, we have used a microscopic theory to study Damon-Eshbach SW modes in the dipole-exchange regime in thin ferromagnetic films. We analyzed the dependence of the DE modes on the SW propagation direction and the ratio between dipolar and exchange strengths. The results show that the DE modes tends to vanish as the system change from the Voigt geometry to a general propagation direction, and that this change depends on the 
strength of the ratio between the dipole and exchange interactions.

We acknowledge the financial support from the FUNCAP, CAPES and CNPq, Brazilian agencies. 


\section{REFERENCES}

[1] N. D. Mermin and H. Wagner, Phys. Rev. Lett. 17, 1133 (1966).

[2] R.W. Damon and J.R. Eshbach, J. Phys. Chem. Solids 19, 308 (1961).

[3] R. P. Erickson, and D. L. Mills, Phys. Rev. B44, 11825 (1991).

[4] F. C. Nörtemann, R. L. Stamps, and R. E. Camley, Phys. Rev. B47, 11910 (1993).

[5] R. N. Costa Filho, M. G. Cottam and G. A. Farias, Solid State Commun. 7, 439 (1998).

[6] D. L. Mills, Surface Excitations, V.M. Agranovich and R. Loudon, pp. 379-438 (1984).

[7] T. Wolfram and R.E. De Wames, Phys. Rev. Lett. 26, 1489 (1970).

[8] M. G. Cottam (ed.), Linear and Nonlinear Spin Waves in Magnetic Films and Superlattices. World Scientific, Singapore, (1994).

[9] D. Kontos and M.G. Cottam, J. Phys. C, B19, 1189 and 1203 (1986). 


\section{FIGURE CAPTIONS}

Fig.1 The linear SW dispersion relation for the lowest branches of (a) a 20-layer $\mathrm{GdCl}_{3}$ film and (b) a 120-layer Fe film. In both cases $\theta=0$.

Fig.2 The SW frequencies as a function of the propagation direction $\theta$ for (a) a 20-layer $\mathrm{GdCl}_{3}$ film taking $q a / \pi=0.02$ and (b) a 120-layer Fe film for $q a / \pi=0.002$. 


\section{FIGURES}
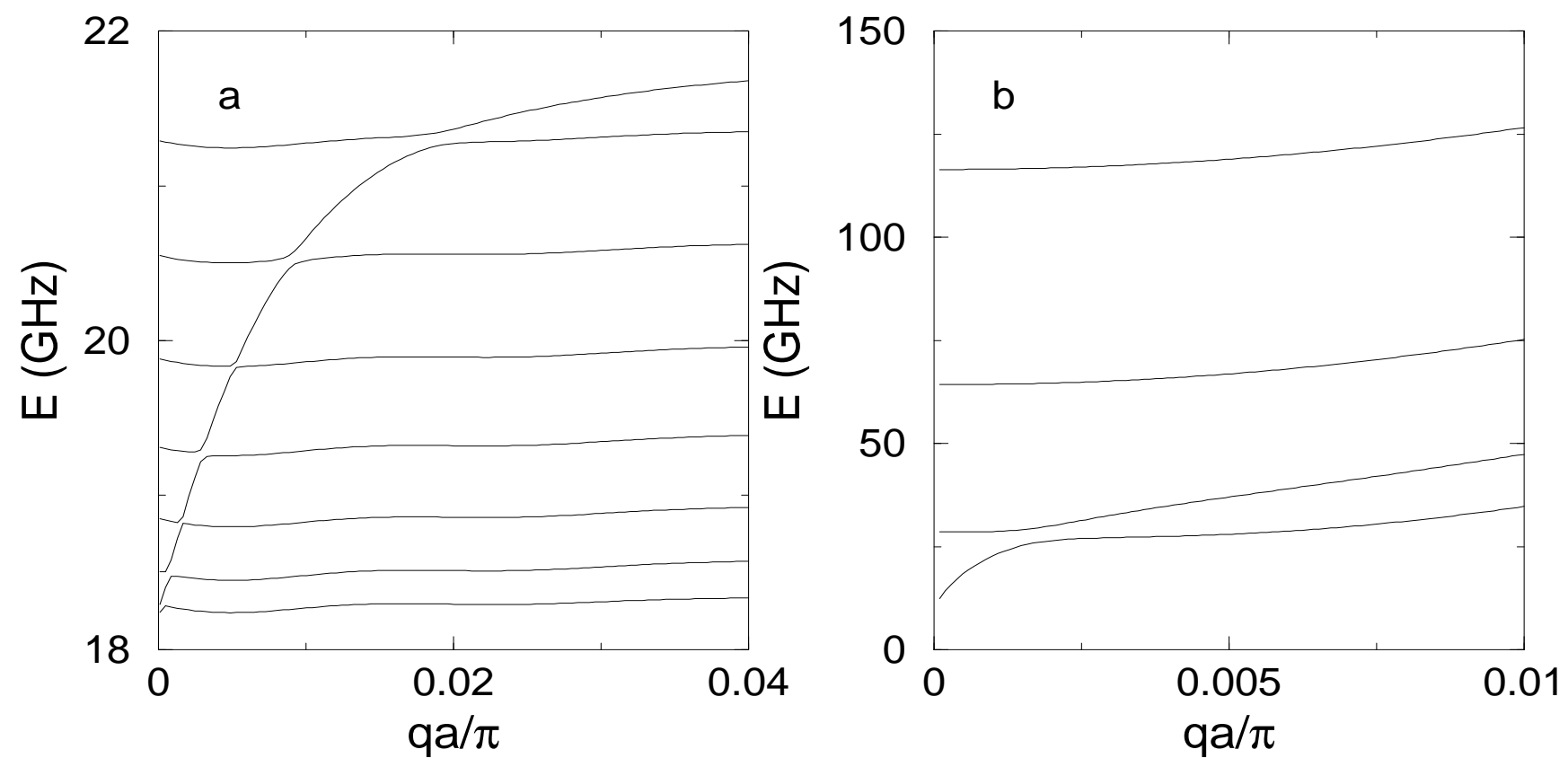

FIG. 1. R.V. Leite at al. 

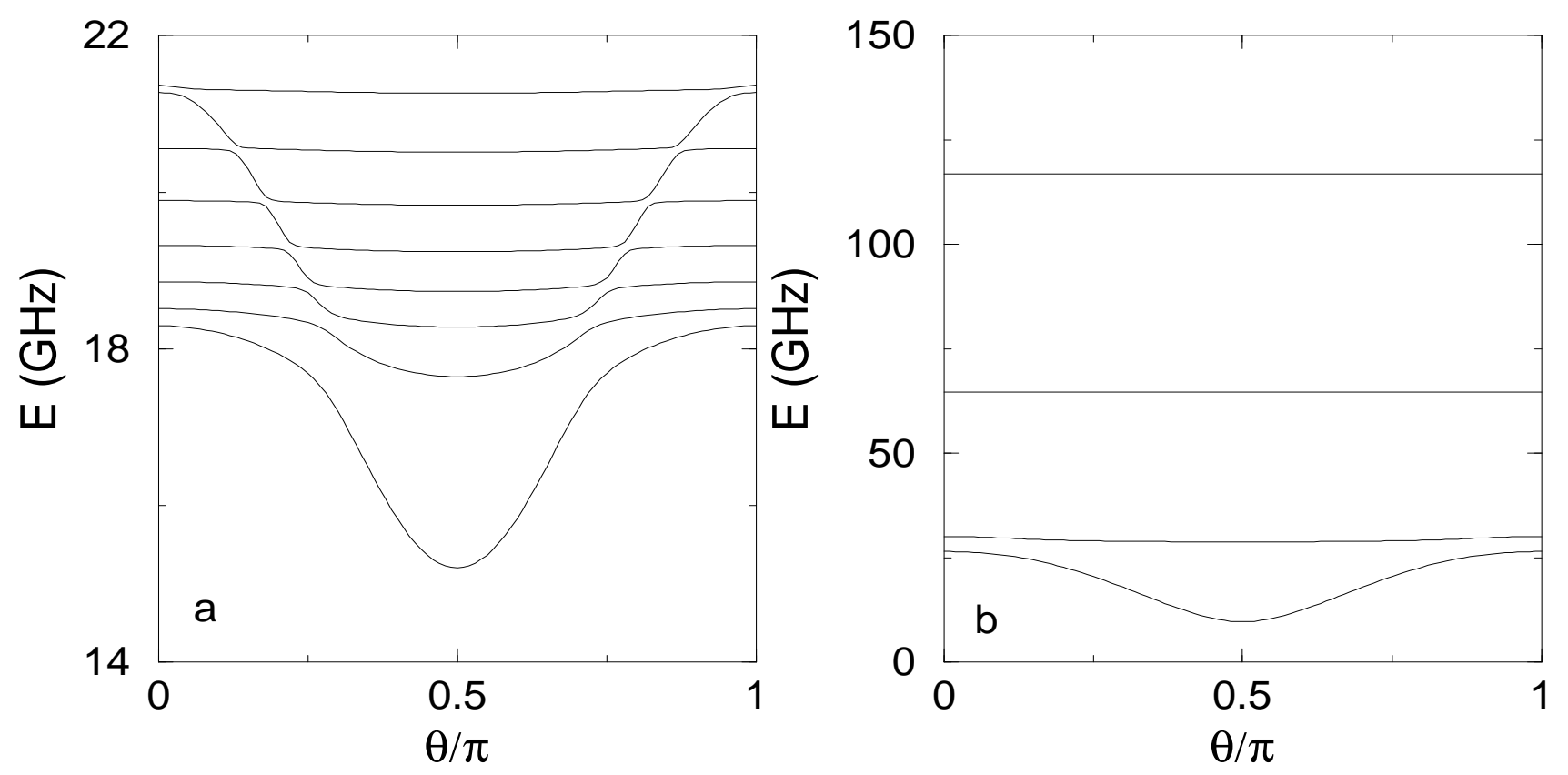

FIG. 2. R.V. Leite at al. 